INPLASY

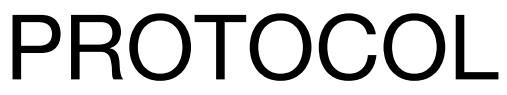

To cite: Yu et al. The acupuncture therapy for patients with postpartum stress urinary incontinence: A protocol for a systematic review and meta analysis. Inplasy protocol 202170050. doi:

10.37766/inplasy2021.7.0050

Received: 16 July 2021

Published: 16 July 2021

Corresponding author:

Huirong Huang

1436682921@qq.com

Author Affiliation:

The Affiliated People's

Hospital of Fujian University of

Traditional Chinese Medicine.

Support: JDZX201920.

Review Stage at time of this submission: The review has not yet started.

Conflicts of interest:

None declared.

\section{The acupuncture therapy for patients with postpartum stress urinary incontinence: A protocol for a systematic review and meta analysis}

Yu, Z1; Huang, H2; Xue, J3; Liu, Q4; Han, X5.

Review question / Objective: The aim of this systematic review is to evaluate whether acupuncture is a more effective and safer therapy for patients with postpartum stress urinary incontinence.

Condition being studied: Postpartum stress urinary incontinence (PSUI) is secondary to pregnancy and childbirth, and involuntary urine leakage from the ureteral orithole occurs when abdominal pressure increases, such as physical activity, sneezing or coughing. Acupuncture therapy is a traditional external treatment of traditional Chinese medicine. In recent years, it has been applied more and more widely in the treatment of PSUI, and has shown good clinical efficacy. However, there is no systematic review of acupuncture therapy for PSUI.

INPLASY registration number: This protocol was registered with the International Platform of Registered Systematic Review and Meta-Analysis Protocols (INPLASY) on 16 July 2021 and was last updated on 16 July 2021 (registration number INPLASY202170050).

\section{INTRODUCTION}

Review question / Objective: The aim of this systematic review is to evaluate whether acupuncture is a more effective and safer therapy for patients with postpartum stress urinary incontinence.
Condition being studied: Postpartum stress urinary incontinence (PSUI) is secondary to pregnancy and childbirth, and involuntary urine leakage from the ureteral orithole occurs when abdominal pressure increases, such as physical activity, sneezing or coughing. Acupuncture 
therapy is a traditional external treatment of traditional Chinese medicine. In recent years, it has been applied more and more widely in the treatment of PSUI, and has shown good clinical efficacy. However, there is no systematic review of acupuncture therapy for PSUI.

\section{METHODS}

Search strategy: The following databases will be searched from the establishment database to July 2021: PubMed, Embase, Web of Science, Cochrane Library, China National Knowledge Infrastructure(CNKI), the Chongqing VIP Chinese Science and Technology Periodical Database (VIP), Wanfang Database, China Biomedical Literature Database(CBM). We will also search grey literature from World Health Organization Clinical Trials Registry, ClinicalTrials.gov and Chinese clinical registry. Reference lists of articles will be retrieved as additional studies. The following search terms will be used: [("Acupuncture therapy" OR "Acupuncture" OR "Electro Acupuncture" OR "Needle" OR "Acupoint Catgut Embedding" OR "Acupoint Injection" OR "Moxibustion") AND ("Postpartum Period" OR "Parturition" OR "Period, Postpartum" OR "Postpartum" OR "Postpartum Women" OR "Women, Postpartum" OR "Puerperium" OR "Parturitions" OR "Birth" OR "Births" OR "Childbirth" OR "Childbirths") AND ("Urinary Incontinence, Stress" OR "Urinary Incontinence" OR "Urinary Stress Incontinence" OR "Incontinence, Urinary Stress" OR "Stress Incontinence, Urinary" OR "Incontinence, Urinary")].

Participant or population: Women with a diagnosis of PSUI will be included and have clear diagnostic criteria, regardless of age, parity, gestational weeks, mode of delivery, duration of disease, race, or nationality.

Intervention: The experimental group was additionally treated with any form of acupuncture therapy in addition to the control group. There are no restrictions on the materials, methods, acupuncture points, intervention time and treatment course of acupuncture.
Comparator: The control group was treated with floor rehabilitation therapy, drug therapy and other non-acupuncture therapy.

Study designs to be included: We will include randomized controlled trials (RCTs) reporting in English or Chinese without any regional restrictions.

Eligibility criteria: (1) The RCTs are eligible, whether or not blind or distributive hiding is specifically described. The language is limited to Chinese and English. (2) If the full article cannot be obtained, the article will be excluded. (3) Articles with statistical errors or incomplete data with no access to the original data will be excluded. (4) If the article is republished, select the one with the most complete data.

Information sources: The following databases will be searched from the establishment database to July 2021: PubMed, Embase, Web of Science, Cochrane Library, China National Knowledge Infrastructure(CNKI), the Chongqing VIP Chinese Science and Technology Periodical Database (VIP), Wanfang Database, China Biomedical Literature Database(CBM). We will also search grey literature from World Health Organization Clinical Trials Registry, ClinicalTrials.gov and Chinese clinical registry. Reference lists of articles will be retrieved as additional studies.

Main outcome(s): The score of International Consultation on Incontinence Questionnaire-short form (ICIQ-SF) and clinical effective rate will be regarded as the primary outcomes.

Additional outcome(s): The pelvic floor muscle potential value and adverse effects will be regarded as the additional outcome.

Data management: All retrieval articles will be imported into the NoteExpress software. Then, two authors will independently select the articles through reviewing the titles, abstracts and full article according to the inclusion criteria and the exclusion criteria. Excluded studies will be listed in a form 
with reasons for their exclusion. The selection results will be cross-checked by two authors. Any divarication between two authors will be solved via discussing and negotiating with the third author firstly, a standard form will be designed for data collection. Then, two authors will independently extract and record the number in strict accordance with the established form. The standard form consists of general information (such as title, the first author, year of publication), details of study (such as design, inclusion and exclusion criteria, diagnostic criteria, blinding, randomization, and sample size), subject information (such as age and numbers), procedures of intervention (such as type of acupuncture, intervention times, frequency), types of outcomes (such as primary, secondary and diverse events), and other detailed information. When there is a disagreement, two authors will discuss and negotiate with the third author to decide. If necessary, we will contact with corresponding authors of the paper to obtain further information.

Quality assessment / Risk of bias analysis: Two authors independently evaluated the risk of bias in the included studies using the bias risk assessment tool recommended by the Cochrane Collaboration in the Cochrane Manual 5.1.0. The tool includes the following seven components: random sequence generation, allocation concealment, blinding of outcome assessors, completeness of outcome data, selective outcome reporting and other biases. The assessments for each item be classified as low risk, high risk, and unclear. If there is a lack of information in the study regarding the risk of bias assessment, corresponding authors of the study will be contacted. When there is a disagreement, two authors will discuss and negotiate with the third author to decide. If the included studies beyond 10, the visual asymmetry of funnel plot will apply to analyze to potential reporting bias. When funnel plot is unclear, we will perform Egger's test and Begg's test by StataMP 16.0 software to quantitative analysis.
Strategy of data synthesis: Review Manager 5.3 software will be performed for data analysis. The continuous outcomes will be indicated the mean difference (MD) with $95 \% \mathrm{Cl}$, while dichotomous data will be expressed as RR with $95 \% \mathrm{Cl}$. When the same outcome measured in different ways, the SMD with $95 \% \mathrm{Cl}$ will be used to express the intervention effect. The ChiSquared test and 12 statistic will be used to assess heterogeneity. If $12<50 \%$, which means the heterogeneity difference is small among the studies included, the fixedeffect model will be adopted. If $12 \geq 50 \%$, which means the heterogeneity difference is significant among the studies included, the random-effect model will be adopted.

Subgroup analysis: If there is significant heterogeneity, we will use StataMP 16.0 software for Meta regression analysis to explore the sources of heterogeneity and conduct subgroup analysis on the factors that may lead to heterogeneity.

Sensitivity analysis: If there is significant heterogeneity, we will use the method of eliminating individual studies one by one to conduct sensitivity analysis, assess the source of heterogeneity, and check the stability of the results.

Language: The language is limited to Chinese and English.

Country(ies) involved: China.

Keywords: acupuncture therapy; stress urinary incontinence; postpartum period; rehabilitation; Meta-analysis.

Contributions of each author:

Author 1 - Zhenling Yu - The author drafted the manuscript.

Email: yzlyen@163.com

Author 2 - Huirong Huang - The author read, provided feedback and approved the final manuscript.

Email: 1436682921@qq.com

Author 3 - Jialu Xue - The author contributed to the development of the selection criteria, and the risk of bias assessment strategy. 
Email: 1105676426@qq.com

Author 4 - Qinyu Liu - The author provided statistical expertise.

Email: 756575679@qq.com

Author 5 - Xueqi Han - The author provided

statistical expertise.

Email: 393877560@qq.com 